\title{
Heparin Use in Hemodialysis Patients following Gastrointestinal Bleeding
}

\author{
Jenny I. Shen ${ }^{a, c}$ Aya A. Mitani ${ }^{b}$ Wolfgang C. Winkelmayer ${ }^{a, d}$ \\ ${ }^{a}$ Division of Nephrology, ${ }^{b}$ Division of General Medical Disciplines, Department of Medicine, Stanford University \\ School of Medicine, Palo Alto, Calif., ' Division of Nephrology and Hypertension, Department of Medicine, Los

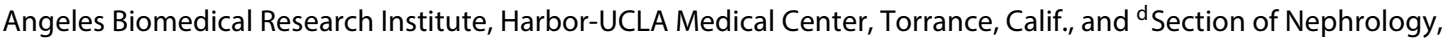 \\ Department of Medicine, Baylor College of Medicine, Houston, Tex., USA
}

\section{Key Words}

Anti-coagulation · Gastrointestinal bleeding ·

Hemodialysis · Heparin

\begin{abstract}
Background: Heparin is commonly given during hemodialysis (HD). Patients undergoing HD have a high rate of gastrointestinal bleeding (GIB). It is unclear whether or when it is safe to give heparin after acute GIB. We describe the patterns and safety of heparin use with outpatient HD following an acute GIB. Methods: We identified patients aged $\geq 67$ who, from 2004-2008, experienced GIB requiring hospitalization within 2 days of receiving maintenance HD with heparin. We used Cox regression to estimate the risk of recurrent GIB and death associated with receiving heparin the day they resumed outpatient HD post-GIB. Results: Of the 1,342 patients who had GIB, 1,158 (86\%) received heparin at a median dose of 4,000 units with their first outpatient $H D$ session after discharge from GIB. On average, their post-GIB doses were slightly lower than their pre-GIB doses (mean change: $-214 \pm 3,266$ units, $p<0.02$ ). However, only $27 \%$ of patients had a decrease in their dose, while $21 \%$ had their dose increased. We did not find an increased risk of death or recurrent GIB associated with using heparin post-GIB (HR; 95\% confidence interval $(\mathrm{Cl})$, for death: $1.01 ; 0.69-1.48$; for recurrent GIB: 0.78 ; 0.39-1.57). Conclusions: The vast majority of these high-risk patients received heparin on the very first
\end{abstract}

day they resumed outpatient HD post-GIB, and the majority at unchanged doses to those received pre-GIB. Even if the practice was not associated with increased risks of death or re-bleeding, it highlights an area for possible system-based improvement to the care for patients on HD.

(C) 2014 S. Karger AG, Basel

\section{Introduction}

Practitioners commonly use heparin to anticoagulate the dialysis circuit during hemodialysis (HD). Between 2004 and 2008, 93\% of older U.S. patients who initiated $\mathrm{HD}$ with a national dialysis provider received heparin during their $\mathrm{HD}$ session [1]. However, patients on $\mathrm{HD}$ have gastrointestinal bleeding events (GIB) at a considerably high rate of 6 events per 100 person-years [2]. Yet, it is unclear whether or when it is safe to restart heparin after a GIB. In this retrospective cohort study, we describe the patterns and safety of heparin use with outpatient HD following discharge from a hospitalization for a GIB.

\section{Materials and Methods}

Data Source

We used data merged from the United States Renal Data System (USRDS) and the electronic medical records (EMR) of a national dialysis provider using a crosswalk of anonymized patient

\section{KARGER}

E-Mail karger@karger.com

www.karger.com/ajn (c) 2014 S. Karger AG, Basel

0250-8095/14/0404-0300\$39.50/0
Jenny I. Shen, MD, MS

Division of Nephrology and Hypertension, Department of Medicine

Harbor-UCLA Medical Center, 1000 W. Carson St., C1 Annex

Torrance, CA 90502 (USA)

E-Mail jshen@labiomed.org 
identifiers that the USRDS Center generated upon approval by the Centers for Medicare and Medicaid Services (CMS) and the National Institutes of Diabetes and Digestive and Kidney Diseases (NIDDK). The USRDS contains demographic information for almost all Americans with end-stage renal disease, billing claims to Medicare (Parts A and B), which are available for the vast majority of Americans from the age of 65, as well as comorbidities and dialysis facility information from forms submitted to the CMS. The EMR includes records on heparin and warfarin use, vital signs, laboratory measurements, and HD prescriptions.

\section{Study Population}

We selected a contemporary cohort of patients who experienced an upper non-variceal GIB (as defined by the stringent criteria in Yang et al. [2]) requiring hospitalization sometime between January 1, 2004 and December 31, 2008. We included only those patients who were admitted within two days of receiving heparin with outpatient HD at a participating facility. This twoday window is to allow time between the onset of symptoms and presentation/admission to the hospital. We excluded patients who did not resume outpatient HD following the GIB. We restricted our study population to patients 67 years of age or older whose primary payer was Medicare for at least one year prior to the index date so that we could uniformly ascertain comorbidities from Medicare claims data. Similarly, patients must have been dialyzing with the provider for at least 30 days prior to the event so that we could ascertain lab values. We also excluded patients with a history of warfarin use in the year prior to the index date as the drug has been shown to increase the risk of hemorrhage in patients on dialysis [3-6]. Please see figure 1 , which outlines the study population selection. Our final cohort included 1,342 patients.

\section{Exposure: Heparin}

We categorized patients as users, or exposed, if they received heparin during their first outpatient HD session following discharge from the hospitalization for GIB. We abstracted data on both the treatment date and heparin administration from the EMR of the dialysis provider. We did not consider any heparin given as catheter lock.

\section{Death and Recurrent GIB}

To evaluate the safety of receiving heparin post-GIB, we estimated its association with death and first recurrent GIB. These events were ascertained from inpatient Medicare claims data and the USRDS death file based on a previously validated algorithm [2].

\section{Other Variables}

Demographics, comorbidities, GIB characteristics, vital signs, laboratory measurements, dialysis characteristics, and facility factors were chosen a priori as potentially and clinically relevant confounders. All variables are listed in table 1.

Demographic factors were obtained from the USRDS. Comorbidity status was derived from both the Medical Evidence Report (CMS form 2728) and Medicare claims data predating the index date by up to one year. The comorbidity definitions, which were derived from both previously validated algorithms and a comprehensive search of ICD-9 diagnosis codes, are provided in online suppl. table 1 (for all online suppl. material, see www.karger.com/ doi/10.1159/000367901). Comorbidities were assigned if coded in at least one inpatient claim or two outpatient claims more than 30

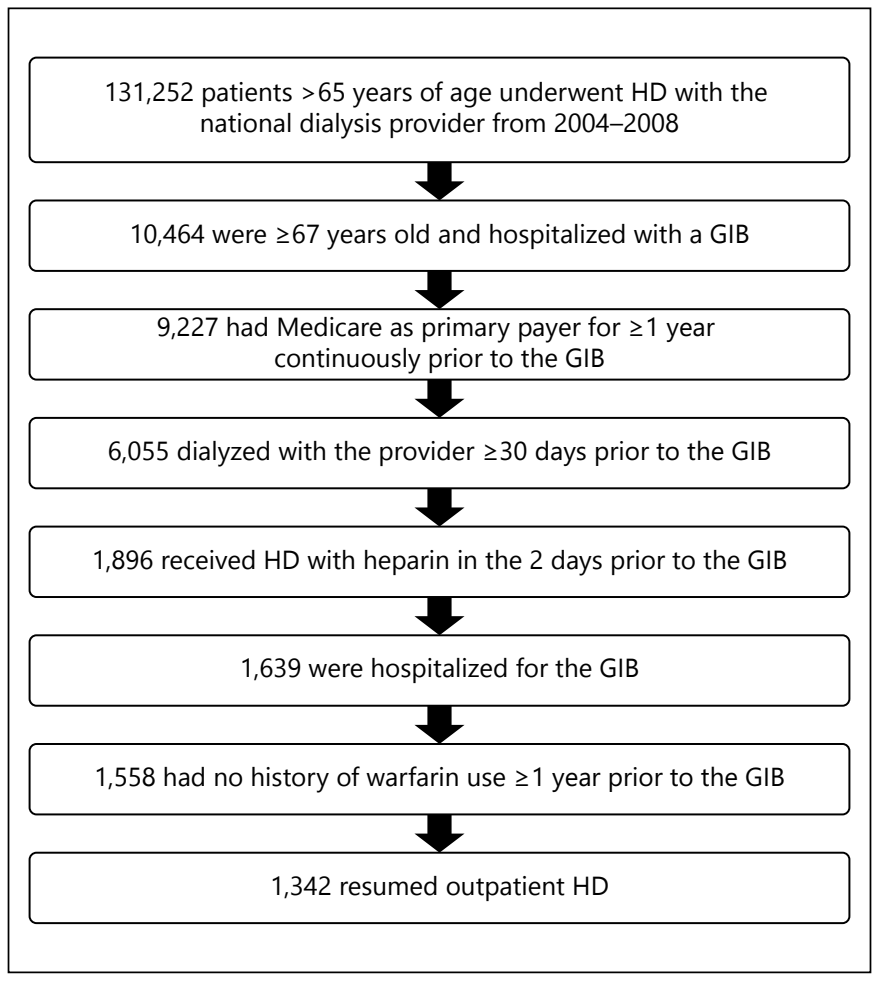

Fig. 1. Study population selection from the United States Renal Data System. We selected a cohort of patients 67 years of age or older whose primary payer was Medicare who, between 2004 and 2008, experienced a gastrointestinal bleeding event requiring hospitalization within two days of receiving heparin with outpatient HD. GIB = Gastrointestinal bleeding event; $\mathrm{HD}=$ hemodialysis .

days apart in the year prior to the index date [2]. GIB characteristics were abstracted from Medicare claims data; the time to restarting outpatient HD was also calculated using EMR data. Vital signs, laboratory measurements, and dialysis characteristics were abstracted from the EMR. Facility factors were taken from USRDS (see table 1 footnotes for more detail). Variables were categorized into clinically relevant ranges, except for weight and facility size, which were categorized by quartile.

\section{Statistical Analysis}

We described baseline characteristics of the cohort using means and standard deviations for normally distributed continuous data, medians and 25th and 75th percentile values for non-normally distributed data, and counts and proportions for categorical data. Differences between those who received heparin post-GIB and those who did not were compared using the Student's $t$ test, Wilcoxon ranksum test, or $\chi^{2}$ test, as appropriate. Paired $t$ test was used to compare heparin doses pre- and post-GIB in those who restarted heparin.

We used proportional hazards regression to estimate the hazard ratio for (1) death and (2) recurrent GIB in those who received heparin post-GIB $v s$. those who did not. We defined the index date as the day that they resumed outpatient HD and followed patients for a year after the index date. We censored patients for change in heparin user status (i.e., when heparin users 
Table 1. Baseline characteristics of patients who resumed outpatient hemodialysis (HD) after a gastrointestinal bleeding event (GIB), categorized by whether they received heparin post-GIB

\begin{tabular}{|c|c|c|c|}
\hline Variable & $\begin{array}{l}\text { Did not receive } \\
\text { heparin post-GIB } \\
(\mathrm{n}=184)\end{array}$ & $\begin{array}{l}\text { Received } \\
\text { heparin post-GIB } \\
(\mathrm{n}=1,158)\end{array}$ & $\mathrm{p}$ value \\
\hline \multicolumn{4}{|l|}{ Demographics } \\
\hline Age, years, mean \pm SD & $78 \pm 6$ & $77 \pm 6$ & 0.02 \\
\hline Male, sex, n (\%) & $91(49)$ & $573(49)$ & 0.99 \\
\hline Race, n (\%) & & & 0.04 \\
\hline Black & $46(25)$ & $372(32)$ & \\
\hline White & $129(70)$ & $701(61)$ & \\
\hline Other ${ }^{1}$ & $-(5)$ & $85(7)$ & \\
\hline Hispanic ethnicity & $20(11)$ & $150(13)$ & 0.43 \\
\hline Years on dialysis (median \& IQR) & $1.7(0.6-3.4)$ & $1.8(0.7-3.5)$ & 0.49 \\
\hline \multicolumn{4}{|l|}{ Reported comorbidities, $n(\%)$} \\
\hline Arrhythmia & $40(22)$ & $238(21)$ & 0.71 \\
\hline Cancer & $15(8)$ & $154(13)$ & 0.05 \\
\hline Coronary artery disease & $82(45)$ & $506(44)$ & 0.83 \\
\hline Deep vein thrombosis ${ }^{1}$ & $-(4)$ & $123(11)$ & 0.01 \\
\hline Diabetes mellitus & $100(54)$ & $673(58)$ & 0.34 \\
\hline Previous gastrointestinal bleeding & $22(12)$ & $140(12)$ & 0.96 \\
\hline Heart failure & $85(46)$ & $576(50)$ & 0.37 \\
\hline Hemorrhagic stroke $^{1}$ & $-(1)$ & $-(1)$ & 0.73 \\
\hline Ischemic stroke & $42(23)$ & $235(20)$ & 0.43 \\
\hline Liver disease $^{1}$ & $-(4)$ & $61(5)$ & 0.40 \\
\hline Peripheral vascular disease & $56(30)$ & $368(32)$ & 0.67 \\
\hline Pulmonary disease & $37(20)$ & $258(22)$ & 0.50 \\
\hline Pulmonary embolism ${ }^{1}$ & $-(<1)$ & $19(2)$ & 0.08 \\
\hline \multicolumn{4}{|l|}{ GIB characteristics } \\
\hline Year GIB occurred & & & 0.35 \\
\hline 2004 & $24(13)$ & $179(15)$ & \\
\hline 2005 & $48(26)$ & $324(28)$ & \\
\hline 2006 & $30(16)$ & $230(20)$ & \\
\hline 2007 & $44(24)$ & $228(20)$ & \\
\hline 2008 & $38(21)$ & $197(17)$ & \\
\hline \multirow{2}{*}{\multicolumn{4}{|c|}{ GIB hospitalization ${ }^{1}$}} \\
\hline & & & \\
\hline 0 & $100(54)$ & $726(63)$ & \\
\hline 1 & $80(43)$ & $424(37)$ & \\
\hline 2 & $-(1)$ & $-(<1)$ & \\
\hline 3 & $-(2)$ & $-(<1)$ & \\
\hline \multicolumn{4}{|l|}{ Days from hospital admission for GIB to } \\
\hline resumption of outpatient $\mathrm{HD}$ (median \& IQR) & $7(5-11)$ & $7(5-12)$ & 0.16 \\
\hline \multicolumn{4}{|l|}{ Vital signs and laboratory measurements } \\
\hline Weight (kg) by quartile & & & 0.29 \\
\hline$<58$ & $46(25)$ & $269(23)$ & \\
\hline $58-67.9$ & $37(20)$ & $283(24)$ & \\
\hline $68-78.9$ & $54(29)$ & $275(24)$ & \\
\hline$\geq 79$ & $40(22)$ & $276(24)$ & \\
\hline Pre-dialysis systolic blood pressure (mm Hg) & & & 0.44 \\
\hline$<120$ & $53(29)$ & $269(23)$ & \\
\hline $120-139$ & $43(23)$ & $288(25)$ & \\
\hline $140-159$ & $45(24)$ & $289(25)$ & \\
\hline$\geq 160$ & $40(22)$ & $284(25)$ & \\
\hline
\end{tabular}


Table 1. (continued)

\begin{tabular}{|c|c|c|c|}
\hline Variable & $\begin{array}{l}\text { Did not receive } \\
\text { heparin post-GIB } \\
(\mathrm{n}=184)\end{array}$ & $\begin{array}{l}\text { Received } \\
\text { heparin post-GIB } \\
(\mathrm{n}=1,158)\end{array}$ & $\mathrm{p}$ value \\
\hline Hemoglobin (g/dl) & & & 0.79 \\
\hline$<9$ & $28(15)$ & $171(15)$ & \\
\hline 9-9.9 & $17(9)$ & $118(10)$ & \\
\hline $10-10.9$ & $26(14)$ & $164(14)$ & \\
\hline $11-11.9$ & $42(23)$ & $223(19)$ & \\
\hline $12-12.9$ & $31(17)$ & $241(21)$ & \\
\hline $13-13.9$ & $22(12)$ & $129(11)$ & \\
\hline$\geq 14^{1}$ & $-(5)$ & $75(6)$ & \\
\hline Platelets $\left(\times 10^{3} / \mu \mathrm{l}\right)$ & & & 0.21 \\
\hline$<150$ & $26(14)$ & $128(11)$ & \\
\hline $150-229$ & $49(27)$ & $398(34)$ & \\
\hline $230-310$ & $47(26)$ & $322(28)$ & \\
\hline $311-400$ & $24(13)$ & $128(11)$ & \\
\hline$>400$ & $12(7)$ & $56(5)$ & \\
\hline Albumin (g/dl) & & & 0.66 \\
\hline$<2.5^{1}$ & $-(2)$ & $32(3)$ & \\
\hline $2.5-2.9$ & $12(7)$ & $113(0)$ & \\
\hline $3-3.4$ & $41(22)$ & $259(22)$ & \\
\hline $3.5-3.9$ & $72(39)$ & $443(38)$ & \\
\hline$\geq 4$ & 35 (19) & $197(17)$ & \\
\hline $\mathrm{Kt} / \mathrm{V}$ & & & 0.02 \\
\hline $0.5-1.1$ & $11(6)$ & $82(7)$ & \\
\hline $1.2-1.5$ & $54(29)$ & $450(39)$ & \\
\hline $1.6-1.9$ & $67(36)$ & $379(33)$ & \\
\hline $2.0-3.0$ & $24(13)$ & $91(8)$ & \\
\hline \multicolumn{4}{|l|}{ Dialysis characteristics } \\
\hline Vascular access & & & 0.28 \\
\hline Central venous catheter & $75(41)$ & $515(44)$ & \\
\hline Fistula or graft & $107(58)$ & $617(53)$ & \\
\hline Length of session (minutes) & & & 0.28 \\
\hline$<180$ & $53(29)$ & $274(24)$ & \\
\hline $180-194$ & $11(6)$ & $97(8)$ & \\
\hline $195-209$ & $63(34)$ & $360(31)$ & \\
\hline $210-224$ & $11(6)$ & $94(8)$ & \\
\hline $225-239$ & $36(20)$ & $283(24)$ & \\
\hline$\geq 240$ & $53(29)$ & $274(24)$ & \\
\hline Reuse of dialysis filter (any) & $97(53)$ & $671(58)$ & 0.18 \\
\hline \multicolumn{4}{|c|}{ Facility characteristics } \\
\hline \multicolumn{4}{|c|}{ Number of outpatient hemodialysis } \\
\hline patients (by quartile) $)^{2}$ & & & 0.27 \\
\hline$<53$ & $36(20)$ & $290(25)$ & \\
\hline $53-78$ & $54(29)$ & $287(25)$ & \\
\hline $79-113$ & $42(23)$ & $290(25)$ & \\
\hline$\geq 114$ & $49(27)$ & $282(24)$ & \\
\hline Rural $^{3}, \mathrm{n}(\%)$ & $29(16)$ & $198(17)$ & 0.65 \\
\hline Census division $^{4}, \mathrm{n}(\%)$ & & & 0.22 \\
\hline East North Central & $22(12)$ & $190(16)$ & \\
\hline East South Central & $-(5)$ & $57(5)$ & \\
\hline Middle Atlantic & $23(13)$ & $99(9)$ & \\
\hline Mountain & $-(5)$ & $50(4)$ & \\
\hline New England & $10(5)$ & $45(4)$ & \\
\hline
\end{tabular}


Table 1. (continued)

\begin{tabular}{lll}
\hline Variable & $\begin{array}{l}\text { Did not receive } \\
\text { heparin post-GIB } \\
(\mathrm{n}=184)\end{array}$ & $\begin{array}{l}\text { Received value } \\
\text { heparin post-GIB } \\
(\mathrm{n}=1,158)\end{array}$ \\
\hline Pacific & $32(17)$ & $188(16)$ \\
South Atlantic & $54(29)$ & $322(28)$ \\
West North Central & $13(7)$ & $67(6)$ \\
West South Central & $12(7)$ & $140(12)$ \\
\hline
\end{tabular}

$\mathrm{SD}=$ Standard deviation; IQR = interquartile range. Variables and the $\mathrm{n}(\%)$ patients who did not receive heparin vs. $\mathrm{n}(\%)$ patients who received heparin with missing data: Weight ${ }^{1}$ : - (4) vs. 55 (5); Pre-dialysis systolic blood pressure ${ }^{1}:-(2)$ vs. 28 (2); Hemoglobin ${ }^{1}:-(5)$ vs. 37 (3); Platelet count: 26 (14) vs. 128 (11); Albumin: 20 (11) vs. 114 (10); Kt/V: 28 (15) vs. 156 (13); Vascular access ${ }^{1}$ : - (1) vs. 26 (2); Facility number of outpatient hemodialysis patients $^{1}$ : - (2) vs. - (1); Facility rural status ${ }^{1}$ : - (0) vs. - (0).

${ }^{1}$ Per federal research regulations, any cell counts less than 10 must not be reported.

${ }^{2}$ Reflects the number of outpatient hemodialysis patients treated at a facility at the end of year the GIB occurred, per the ESRD Facility Survey (form CMS-2744).

${ }^{3}$ Facilities were considered urban if they were classified as a metropolitan area in the Rural-Urban Commuting Area (RUCA) Codes version 2.0, which are based on 2000 Census commuting data and 2004 zip codes; all other areas were considered to be rural [10].

${ }^{4}$ Facilities were categorized into one of nine US Census Bureau Divisions based on their state [11].

stopped receiving heparin and when non-users started using heparin), kidney transplantation, end of the follow-up period (December 31, 2010), and death (for the recurrent GIB analysis). As patients may have had multiple recurrent GIB, we only analyzed the first event they experienced. Violation of the proportional hazards assumption was checked using interaction terms with time.

We ran both unadjusted and adjusted analyses. We tested two models that were adjusted for the following:

(1) all variables that differed between the groups at a significance level of $\mathrm{p}<0.20$ (chosen to include more potentially confounding factors than the more restrictive p level of 0.05 )

(2) model $1+$ all demographic factors, all comorbidities, hemoglobin concentration, platelet count, and vascular access type.

We ran both models for death. For recurrent GIB, we only ran model 1 because the limited number of events would have led to overfitting of model 2. Missing data were imputed using multiple imputations.

\section{Sensitivity Analyses}

Given the potential for confounding in this observational study, we performed a number of sensitivity analyses. First, we estimated an unadjusted hazard ratio for death and recurrent GIB excluding events that happened within 14 days of the index date, as these events may have simply been an extension of the index GIB. We moved the index date from the first day that the patients resumed outpatient dialysis to the $14^{\text {th }}$ day that they were on outpatient dialysis, thus excluding from analysis anyone who had a recurrent bleed, who died, or who changed exposures during these first 14 days.

To address the potential selection bias, we also repeated an unadjusted analysis restricting the population to patients for whom GIB was listed as the primary diagnosis for the hospitalization (vs. any diagnosis in the primary analysis) because GIB listed as secondary diagnoses may not be as severe.

Given the inherent risk of confounding by indication, we further conducted propensity score adjusted analyses. Using a logistic regression model that included all of the variables listed in table 1 , we generated propensity scores for receiving heparin post-GIB. We then performed the following Cox regressions for both death and recurrent GIB:

(1) stratified by quintile of propensity score

(2) adjusted for propensity score as a continuous variable

(3) model 2 , but excluding the $5 \%$ of the cohort with the most extreme propensity scores (i.e., those with the top and bottom $2.5 \%$ of propensity scores).

Finally, to address the censoring bias, we performed an unadjusted analysis in which we did not censor for change in exposure. This is analogous to an intention-to-treat analysis. Similarly, we also completed an unadjusted analysis in which we censored patients two days after a change in exposure (vs. on the day of the change in the exposure), as not to miss the events that may have been the result of the original exposure.

All statistical tests were two-tailed. p values $<0.05$ were considered statistically significant, and we did not adjust for multiple comparisons. All analyses were performed using SAS Enterprise Guide 4.3 (SAS Institute Inc., Cary, NC, USA).

\section{Results}

Out of 1,342 patients, 1,158 (86\%) received heparin with their first outpatient HD session. These post-GIB heparin users were slightly younger, more likely to be black, and 


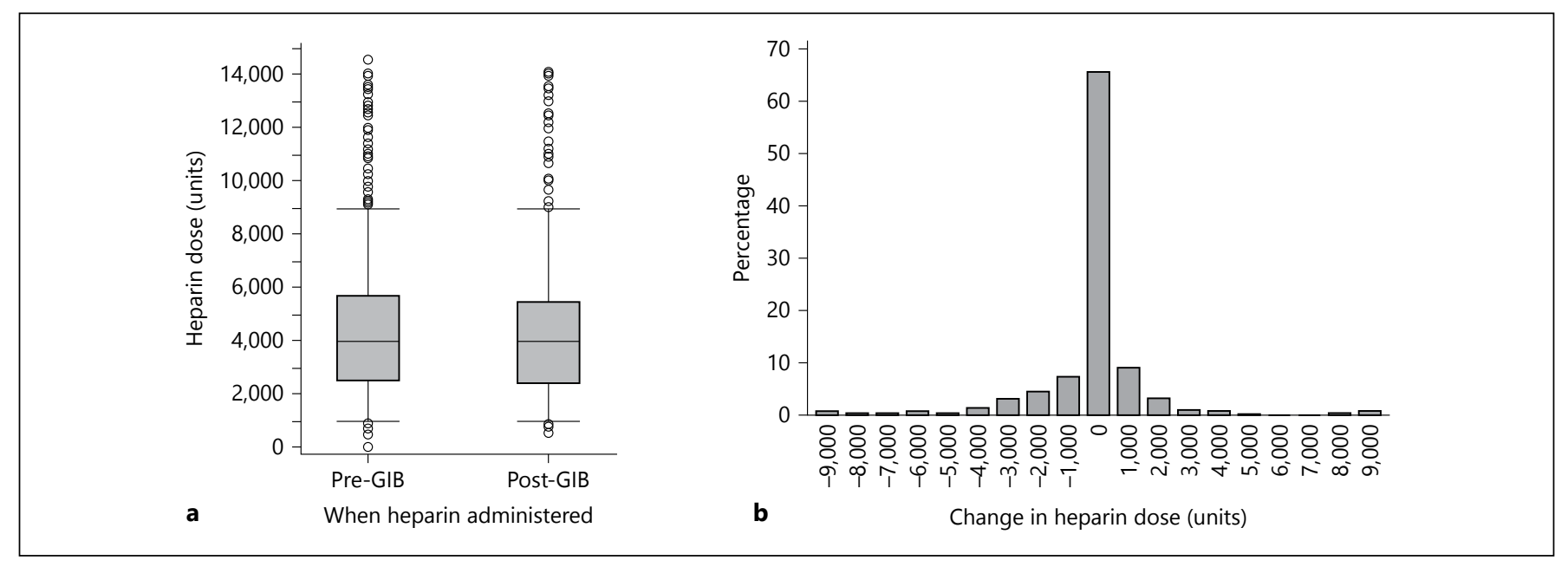

Fig. 2. Panel A: Comparison of heparin doses given for outpatient hemodialysis (HD) pre- vs. post-gastrointestinal bleeding (GIB). Whisker edges represent the 5th and 95th percentiles. Panel B: Distribution of the change in heparin dose from pre- to post-GIB. Xaxis labels are the midpoints for the range of values represented in that bar. For example, the ' 0 ' bar represents patients whose change in heparin dose range from -500 units to 500 units. Population for both panels restricted to patients who received heparin in the first session that they resumed outpatient HD $(n=1,158)$. more likely to have a history of cancer or deep vein thrombosis (table 1). Otherwise, they were similar to those who did not restart heparin, with comparable comorbidities, vital signs, laboratory measurements, and dialysis and facility characteristics. Although post-GIB heparin users had received blood transfusions on fewer days during their hospitalization, they had taken just as many days to return to outpatient HD as those who did not receive heparin post-GIB.

Patients given heparin post-GIB received a median dose of 4,000 units post-GIB (interquartile range: 2,5005,600 units) (fig. 2). On average, their post-GIB doses were lower than their pre-GIB doses (mean change in heparin dose: $-214 \pm 3,266$ units, $\mathrm{p}<0.02$ ). However, only $27 \%$ of patients had a decrease in their dose, and nearly the same proportion, $21 \%$, had their dose increased.

Over 694 and 661 person-years of follow-up, we observed 420 deaths and 90 recurrent GIB events for a rate of 61 deaths and 14 recurrent GIB per 100 person-years, respectively (table 2). We did not find any significant associations between heparin administration post-GIB and death [hazard ratio (HR) and 95\% Confidence Interval (CI): $1.01(0.69-1.48)$ ] or recurrent GIB [HR and 95\% CI: $0.78(0.39-1.57)]$.

The follow-up time for those who did not receive heparin post-GIB was shorter than for those who did, because many non-users of heparin were censored early when they started receiving heparin once again. For non-users, the median time from the first outpatient HD session to receipt of heparin was 7 days [interquartile range (IQR): 3-21 days]. By contrast, for post-GIB heparin users, median time from the first outpatient HD session to the first session without heparin was 79 days (IQR: 16-183 days). However, the HR from the primary analysis did not materially change in adjusted models or in a sensitivity analysis in which we did not censor for crossover between heparin use and non-use (tables 2 and 3). Furthermore, the use of proportional hazards regression takes into account differences in follow-up times. We checked that our models did not violate the proportional hazards assumption, so the estimated HR did not change over the course of the study period despite the difference in median follow-up time between the two groups. HR were also similar in the other sensitivity analyses, including one that excluded events that may have been attributable to the index GIB rather than resumption of outpatient heparin, another that addressed potential selection bias by restricting the cohort to patients for whom GIB was the primary diagnosis for hospitalization, and an analysis that addressed confounding by indication via propensity score adjustment (table 3 ).

\section{Discussion}

This study shows that US practitioners do not have a systematic approach to prescribing heparin following a GIB to reduce the risk of bleeding in this high-risk group. 
Table 2. Number of events, follow-up time, incidence rates, and hazard ratios for death and recurrent gastrointestinal bleeding (GIB) in patients receiving heparin following GIB

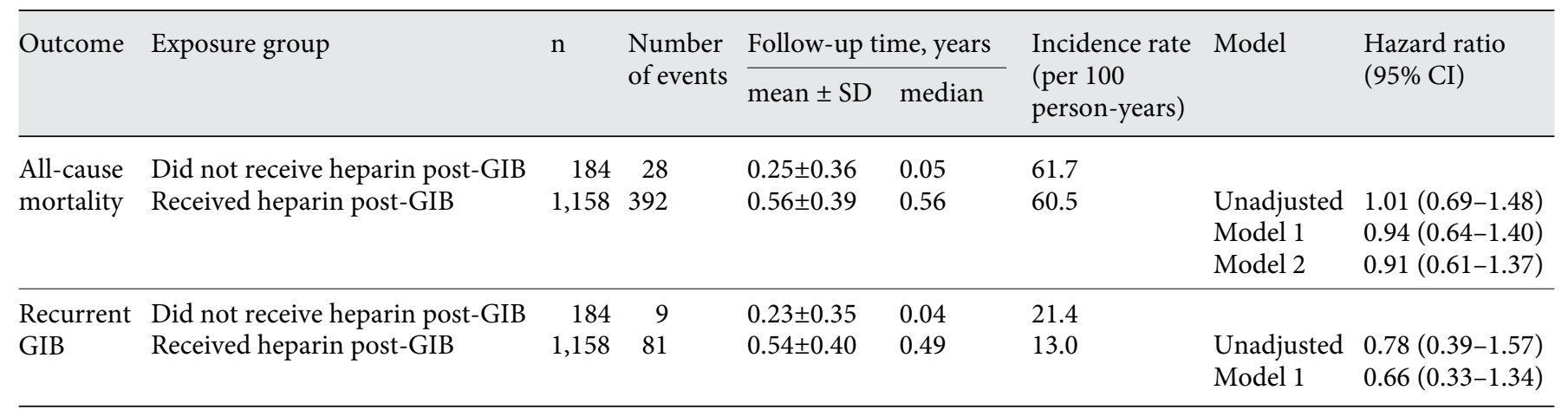

$\mathrm{SD}=$ Standard deviation; $\mathrm{CI}=$ confidence interval.

Model 1: adjusted for all variables that were significantly different between the groups in univariate analysis at the $\mathrm{p}<0.20$ level (age, race, cancer, deep vein thrombosis, pulmonary embolism, transfusions received during hospitalization, days from hospital admission for GIB to resumption of outpatient $\mathrm{HD}, \mathrm{Kt} / \mathrm{V}$, reuse of dialysis filter).

Model 2: Model 1 + all demographics (age, sex, race, ethnicity, years on dialysis), all comorbidities (arrhythmia, cancer, coronary artery disease, deep vein thrombosis, diabetes mellitus, previous gastrointestinal bleeding, heart failure, hemorrhagic stroke, ischemic stroke, liver disease, peripheral vascular disease, pulmonary disease, pulmonary embolism), hemoglobin concentration, platelet count, and access type.

Table 3. Hazard ratios (HR) for death and recurrent gastrointestinal bleeding (GIB) for patients who received heparin vs. those who did not receive heparin following a GIB, based on various sensitivity analyses

\begin{tabular}{|c|c|c|c|c|c|}
\hline Sensitivity Analysis & $\mathrm{n}$ & $\begin{array}{l}\text { Number } \\
\text { of deaths }\end{array}$ & $\begin{array}{l}\text { HR for mortality } \\
\text { (95\% confidence } \\
\text { interval) }\end{array}$ & $\begin{array}{l}\text { Number of } \\
\text { recurrent } \\
\text { GIB }\end{array}$ & $\begin{array}{l}\text { HR for recurrent } \\
\text { GIB ( } 95 \% \text { confidence } \\
\text { interval) }\end{array}$ \\
\hline \multicolumn{6}{|l|}{ Restricted to hospitalizations with GIB as } \\
\hline primary diagnosis & 824 & 230 & $0.92(0.57-1.49)$ & 59 & $0.75(0.34-1.68)$ \\
\hline \multicolumn{6}{|l|}{$\begin{array}{l}\text { Adjusted for propensity score as a continuous variable, } \\
\text { and excluding the top and bottom } 2.5 \% \text { of propensity }\end{array}$} \\
\hline scores $^{2}$ & 1,342 & 420 & $0.88(0.59-1.32)$ & 90 & $0.66(0.32-1.36)$ \\
\hline Not censoring for change in exposure ${ }^{3}$ & 1,342 & 563 & $1.27(0.98-1.64)$ & 113 & $1.01(0.60-1.72)$ \\
\hline Censoring two days after a change in exposure & 1,342 & 421 & $1.01(0.69-1.49)$ & 90 & $0.78(0.39-1.57)$ \\
\hline
\end{tabular}

${ }^{1} \mathrm{~N}$ is $<1,342(\mathrm{n}=105$ patients who did not receive heparin and 1,041 heparin users) because patients who died in the first 14 days were excluded from the analyses.

2 The propensity score (propensity of receiving heparin post-GIB) was generated using all of the variables listed in table 1. C-statistic for the model was 0.72 . Propensity score matching was not possible as the number of heparin users was greater than the number of those who did use heparin post-GIB.

${ }^{3}$ Median follow-up time was 1.00 year for both the group who did not receive heparin and the group who did.

The vast majority of patients received heparin on the very first day they resume outpatient $\mathrm{HD}$, and only at slightly lower doses than they received prior to the GIB; in fact, a fifth even received higher doses. This practice is inconsistent with the conventional wisdom of giving less heparin to patients who have a high risk of bleeding [7]. While it is possible that some of the physicians of these patients made a deliberate decision not to decrease their heparin doses, it is much more likely that most of the physicians were not even aware their patients had experienced a GIB 
or were returning to outpatient HD. Such lack of communication between inpatient and outpatient physicians is common and can compromise patient safety [8]. Although we did not find a significant association between using heparin post-GIB and adverse events, our study was only powered to detect an increase of more than $48 \%$ in the risk of death and $57 \%$ in the risk of recurrent GIB. Thus, we certainly may have missed a clinically meaningful risk to receiving heparin following a GIB.

At the very least, our study suggests that the current system of care for dialysis patients can be improved. Programs that optimize handoffs between hospital-based and outpatient practitioners and systems that trigger the deliberate re-evaluation of a heparin dose following a clinical event rather than automatically resuming the previous dose could help prioritize patient safety. Such programs do not necessarily have to result in the automatic cessation or reduction of heparin. However, they should engage the practitioners and ensure that the physicians consider recent clinical events and exercise clinical judgment when resuming heparin. Such programs could be implemented through the use of information technology, which is already integrated into much of dialysis care [9].

Limitations of this study include residual confounding by indication. Since patients in this observational study were not randomly allocated to receive heparin after the index GIB, those at a higher risk of bleeding may have been less likely to be prescribed heparin. In other words, the heparin non-users may have inherently been at a higher risk of re-bleeding and death, thereby creating a bias in the results causing harm for heparin non-users. We adjusted for as many factors as possible that might affect the decision to give heparin, including age, multiple comorbidities, and thrombocytopenia. However, there are a number of unobserved factors, including the dose of heparin given for HD during the hospitalization and administration of drugs such as aspirin and clopidogrel, which may have confounded the results. Finally, the population was restricted to older Medicare recipients dialyzing with one particular dialysis provider, which may limit the generalizability of our study. These limitations must be weighed against the strengths of the study: use of a large, national, contemporary cohort.

In conclusion, our study shows that heparin is widely, but not systematically, administered in patients who resume outpatient HD following a GIB. Even if the practice is not associated with an increased risk of death or bleeding, it highlights an area for possible system-based improvement to the care for patients on HD.

\section{Acknowledgements}

This study was conducted under a data use agreement between the NIDDK and Dr. Winkelmayer. The manuscript was reviewed by an NIDDK officer and approved for publication. The data reported here have been supplied by the United States Renal Data System and by DaVita, Inc. The interpretation and reporting of these data are the responsibility of the author(s) and in no way should be seen as an official policy or interpretation. This work was supported by grants F32DK096765 and 1R21DK077336 from the National Institute of Diabetes, Digestive, and Kidney Diseases (NIDDK) to Drs. Shen and Winkelmayer. Dr. Shen was also supported by the Clinical Scientist in Nephrology Fellowship from the American Kidney Fund, the Satellite Dialysis Clinical Investigator Award from the National Kidney Foundation grant KL2TR000122 from the NIH/National Center for Advancing Translational Science (NCATS), DaVita Clinical Research, and an anonymous donor.

\section{Disclosure Statement}

The authors have no conflicts of interest to disclose.

\section{References}

1 Shen JI, Winkelmayer WC: Use and safety of unfractionated heparin for anticoagulation during maintenance hemodialysis. Am J Kidney Dis 2012;60:473-486.

$\checkmark 2$ Yang JY, Lee TC, Montez-Rath ME, et al: Trends in acute nonvariceal upper gastrointestinal bleeding in dialysis patients. J Am Soc Nephrol 2012;23:495-506.

>3 Holden RM, Harman GJ, Wang M, Holland D, Day AG: Major bleeding in hemodialysis patients. Clin J Am Soc Nephrol 2008;3:105-110.

4 Chan KE, Lazarus JM, Thadhani R, Hakim RM: Warfarin use associates with increased risk for stroke in hemodialysis patients with atrial fibrillation. J Am Soc Nephrol 2009;20:2223-2233.
55 Winkelmayer WC, Liu J, Setoguchi S, Choudhry NK: Effectiveness and safety of warfarin initiation in older hemodialysis patients with incident atrial fibrillation. Clin J Am Soc Nephrol 2011;6:2662-2668.

6 Wizemann V, Tong L, Satayathum S, et al: Atrial fibrillation in hemodialysis patients: clinical features and associations with anticoagulant therapy. Kidney Int 2010;77:10981106.

7 Davenport A: What are the anticoagulation options for intermittent hemodialysis? Nat Rev Nephrol 2011;7:499-508.

8 Kripalani S, LeFevre F, Phillips CO, Williams MV, Basaviah P, Baker DW: Deficits in com- munication and information transfer between hospital-based and primary care physicians: implications for patient safety and continuity of care. JAMA 2007;297:831-841.

$\checkmark 9$ Bates DW, Gawande AA: Improving safety with information technology. N Engl J Med 2003;348:2526-2534.

10 Rural Urban Commuting Area Codes Data. 2012. http://depts.washington.edu/uwruca/ ruca-data.php (5 January 2012, date last accessed).

11 Census Bureau Regions and Divisions with State FIPS Codes. 2012. http://www.census. gov/geo/www/reg_div.txt (5 January 2012, date last accessed). 